\title{
AVALIAÇÃO DA ADIÇÃO DE RESÍDUO DO CORTE DE ROCHAS ORNAMENTAIS EM MASSA CERÂMICA UTILIZADA NA INDÚSTRIA DA CERÂMICA VERMELHA
}

Lorenna Lúcia Bastos Bandeira ${ }^{1}$

Felipe Casoli Cassimiro ${ }^{2}$

Ailana Kröhling Uliana ${ }^{2}$

Carla Therezinha Dalvi Borjaille Alledi²

André Gustavo de Sousa Galdino ${ }^{2 *}$

\section{RESUMO}

O objetivo desse estudo foi avaliar as propriedades físicas e mecânicas de traços com 0\% (traço de referência), 10\%, 20\%, 30\%, 40\% e 50\% em massa de incorporação de resíduo de corte de rochas ornamentais em massas cerâmicas utilizadas na fabricação de cerâmica estrutural. Foram confeccionados corpos de prova com dimensões de $12 \mathrm{~mm}$ de diâmetro por $24 \mathrm{~mm}$ de altura, sinterizados a $850^{\circ} \mathrm{C}, 900^{\circ} \mathrm{C}$ e $950^{\circ} \mathrm{C}$ e avaliadas as propriedades retração linear de queima, absorção de água, porosidade aparente, massa específica aparente e limite de resistência à compressão. Os resultados obtidos foram comparados com o traço de referência a fím de classificá-los como alternativa viável e passível de utilização para cerâmica estrutural. Conclui-se que é possível adicionar até $30 \%$ esse resíduo em massas para cerâmica estrutural.

Palavras-chave: resíduo de corte de rochas ornamentais, argila estrutural, propriedades físicas e mecânicas.

\section{EVALUATION OF THE ADDITION OF GRANITE CUTTING WASTE USED FOR RED CLAY INDUSTRY}

\begin{abstract}
This work aimed to evaluate physical and mechanical properties of samples with $0 \%$ (reference sample), $10 \%, 20 \%, 30 \%, 40 \%$ and $50 \%$ by mass of natural stone cutting waste addition to ceramic masses used in structural ceramics. Sample parts with $12 \mathrm{~mm}$ diameter and $24 \mathrm{~mm}$ height were sintered at $850^{\circ} \mathrm{C}, 900^{\circ} \mathrm{C}$ and $950^{\circ} \mathrm{C}$ and firing linear shrinkage, water absorption, apparent porosity,

\footnotetext{
${ }^{1}$ Instituto Federal de Ensino, Pesquisa e Extensão do Espírito Santo, Campus São Mateus

${ }^{2}$ Instituto Federal de Ensino, Pesquisa e Extensão do Espírito Santo, Campus Vitória

*Autor para correspondência: andregsg@ifes.edu.br

Manuscrito submetido em: 02 / 09 / 2018

Aceito para publicação em: 14/10/2018

Revista Ifes Ciência, v.4, n.1, 2018 - Instituto Federal do Espírito Santo
} 
apparent density and compressive strength were evaluated. Results were compared to reference sample in order to classify them as viable and usable alternative for structural ceramics. It is concluded that it is possible to add this residue in ceramic masses for structural ceramics up to $30 \%$ by mass.

Key-words: Natural stone cutting waste, structural clay, physical and mechanical properties.

\section{INTRODUÇÃO}

O estado do Espírito Santo é um dos estados produtores de rochas ornamentais, em particular, mármore e granito, com essa atividade sendo desenvolvida de norte ao sul do estado. Em termos nacionais, o Espírito Santo é líder absoluto na produção nacional de rochas, com aporte ao estado de 90\% dos investimentos do parque industrial brasileiro nesse seguimento. A região norte do Espírito Santo possui geologia que garante a formação de granitos de cores diversas (amarelo, bege, branco, preto, verde, etc.) (SARDOU FILHO et al., 2013). Entretanto, a produção de mármore e granito gera resíduos provenientes dos rejeitos das minas ou das unidades de processamento ou dos processos de lixamento e polimento na forma de lama (água utilizada para resfriar e lubrificar as máquinas de lixamento e polimento juntamente com partículas dessas rochas ornamentais) (ALMEIDA et al., 2007).

No mundo, somente em 2007, a indústria de rochas ornamentais apresentou uma produção anual de $68 \mathrm{t}$ de produto processado e no Brasil; em 2005, a geração anual de resíduo fino proveniente dessa produção foi de 800.000 t (ALMEIDA et al., 2007; LIRA, LIRA, NEVES, 2016). Uma vez que o resíduo gerado pelas rochas ornamentais contém grandes quantidades de $\mathrm{SiO}_{2}$ e $\mathrm{Al}_{2} \mathrm{O}_{3}$, vários trabalhos têm sido realizados com esse resíduo, direcionando sua utilização para vitrocerâmicas (KANG et al., 2017), para corretivo e fertilizante de solos agrícolas (COSTA et al., 2010), concreto de vários tipos (ALMEIDA et al., 2007; ALMEIDA, BRANCO, SANTOS, 2007; VIJAYALAKSHMI, SEKAR, GANESH PRABHU, 2013; SINGH, NAGAR, AGRAWAL, 2016a; SINGH et al., 2016a; SINGH et al., 2016b; 11, SADEK, EL-ATTAR, ALI, 2016; RANA et al., 2016), argamassas e sistemas pozolânicos [MENEZES et al., 2009; MÁRMOL et al., 2010; LIRA, LIRA, NEVES, 2016; MEDINA et al., 2017), cerâmica vermelha (VIEIRA et al., 2004; TORRES et al., 2009; HOJAMBERDIEV, EMINOV, XU, 2011), síntese de geopolímeros (TCHADJIÉ et al., 2016), cimento de oxicloreto de magnésio volumétrico (LI et al., 2013), agregados leves (SOLTAN et al., 2016), substituto de areia [SINGH, NAGAR, AGRAWAL, 2016b; SINGH et al., 2016c; GHANNAMA, NAJM, VASCONEZ, 2016), blocos de construção (LOKESHWARI, JAGADISH, 2016; SOUZA et al., 2017). 
O objetivo desse estudo foi avaliar as propriedades físicas e mecânicas de traços com 0\% (traço de referência), 10\%, 20\%, 30\%, 40\% e 50\% em massa de incorporação de resíduo de corte de rochas ornamentais em massas cerâmicas utilizadas na fabricação de cerâmica estrutural.

\section{MATERIAIS E MÉTODOS}

\section{Materiais}

A argila utilizada nesse trabalho foi gentilmente doada pela empresa Cerâmica Lubiana Ltda., que está localizada em Nova Venécia, ES. A argila como recebida foi submetida à fluorescência de raios X (FRX) para determinação da composição química.

O resíduo de corte de rochas ornamentais foi gentilmente doado pelo Centro de Tratamento de Resíduos Nova Venécia S/A, localizada em Nova Venécia, ES. Assim como a argila, o resíduo também foi submetido à FRX para determinação da composição química.

\section{Métodos}

A argila e o resíduo de corte de rochas ornamentais foram beneficiados através de destorroamento e peneiramento em peneira $A B N T n^{\circ} .80(0,177 \mathrm{~mm})$. Foi observado que a massa total do resíduo de corte de rochas ornamentais passou pela abertura da peneira, fato que não aconteceu com a argila, indicando que as partículas do resíduo são menores que os da argila, que teve parte da massa retida. Em seguida, se determinou a quantidade em massa que seria adicionado à argila para esse estudo, sendo analisados seis traços: 0\% (traço de referência - AR), 10\% (A10), 20\% (A20), 30\% (A30), $40 \%$ (A40) e 50\% (A50) em massa de resíduo. As temperaturas de sinterização a que esses traços foram submetidos na forma de corpos de prova cilíndricos foram $850^{\circ} \mathrm{C}, 900^{\circ} \mathrm{C} \mathrm{e} 950^{\circ} \mathrm{C}$, por se tratar das temperaturas utilizadas por boa parte das empresas cerâmicas do ramo de cerâmica vermelha para sinterizar seus produtos.

Corpos de prova cilíndricos com dimensões de $12 \mathrm{~mm}$ de diâmetro por $20 \mathrm{~mm}$ de altura foram confeccionados por prensagem uniaxial com carga de $20 \mathrm{kN}$. Foram confeccionados quinze corpos de prova por cada traço para cada temperatura. Os corpos de prova foram submetidos a ensaios físicos de umidade de prensagem, retração linear de queima, absorção de água, massa específica aparente e porosidade aparente e ao ensaio mecânico de compressão para determinação do limite de resistência à compressão. $\mathrm{O}$ ensaio de compressão foi escolhido devido à atividade fim da empresa Adélio Lubiana Ltda., que é a fabricação de blocos estruturais em cerâmica.

Os valores de retração linear de queima (RLQ), absorção de água (AA), massa específica aparente (MEA), porosidade aparente (PA) e limite de resistência à compressão (LRC) foram obtidos pela 
média aritmética de quinze corpos de prova utilizando-se as equações (1), (2), (3), (4) e (5), respectivamente.

$R L Q(\%)=\left(\frac{L_{0}-L_{1}}{L_{0}}\right) \cdot 100$

$A A(\%)=\left(\frac{m_{u}-m_{s}}{m_{s}}\right) \cdot 100$

$\operatorname{MEA}\left(\frac{\mathrm{g}}{\mathrm{cm} m^{3}}\right)=\frac{m_{u}}{m_{s}-m_{i}}$

$P A(\%)=\left(\frac{m_{u}-m_{s}}{m_{u}-m_{i}}\right) \cdot 100$

$\operatorname{LRC}(M P a)=\frac{F}{S}$

Onde $m_{1}$ é massa do corpo de prova verde antes da prensagem (g), $m_{2}$ é a massa do corpo de prova verde após secagem em estufa $(\mathrm{g}), L_{0}$ é o comprimento inicial do corpo de prova após sinterização $(\mathrm{mm}), L_{1}$ é o comprimento final do corpo de prova após sinterização $(\mathrm{mm}), m_{u}$ é a massa úmida do corpo de prova sinterizado $(\mathrm{g}), m_{s}$ é a massa seca do corpo de prova sinterizado $(\mathrm{g}), m_{i}$ é a massa imersa do corpo de prova sinterizado (g), $F$ é a carga aplicada ao corpo de prova sinterizado (N) e $S$ é a área de aplicação de carga no corpo de prova sinterizado $\left(\mathrm{mm}^{2}\right)$.

\section{RESULTADOS E DISCUSSÃO}

\section{Composição química}

A Tabela 1 apresenta os resultados de composição química da argila e do resíduo de corte de rochas ornamentais.

Tabela 1 - Composição química da argila e do resíduo de corte de rochas ornamentais.

\begin{tabular}{ccc}
\hline & \multicolumn{2}{c}{ Quantidade percentual em massa do material (\%) } \\
\cline { 2 - 3 } Composto & Argila & $\begin{array}{c}\text { Resíduo de corte de rochas } \\
\text { ornamentais }\end{array}$ \\
\hline $\mathrm{SiO}_{2}$ & 52,233 & 68,305 \\
\hline $\mathrm{Al}_{2} \mathrm{O}_{3}$ & 34,738 & 15,763 \\
\hline $\mathrm{K}_{2} \mathrm{O}$ & 2,363 & 4,312 \\
\hline $\mathrm{CaO}$ & $n / d$ & 3,275 \\
\hline $\mathrm{Fe}_{2} \mathrm{O}_{3}$ & 7,315 & 3,223 \\
\hline $\mathrm{Na}_{2} \mathrm{O}$ & $\mathrm{n} / \mathrm{d}$ & 2,909 \\
\hline $\mathrm{SO}_{3}$ & 0,545 & 0,634 \\
\hline $\mathrm{P}_{2} \mathrm{O}_{5}$ & $n / d$ & 0,614 \\
\hline
\end{tabular}




\begin{tabular}{ccc}
\hline $\mathrm{MgO}$ & 1,435 & 0,543 \\
\hline $\mathrm{TiO}_{2}$ & 0,832 & 0,353 \\
\hline $\mathrm{MnO}$ & 0,063 & 0,033 \\
\hline $\mathrm{SrO}$ & $\mathrm{n} / \mathrm{d}$ & 0,021 \\
\hline $\mathrm{Rb}_{2} \mathrm{O}$ & 0,015 & 0,014 \\
\hline $\mathrm{BaO}$ & 0,425 & $\mathrm{n} / \mathrm{d}$
\end{tabular}

n/d - não determinado.

Observa-se que a argila é composta majoriatariamente por $\mathrm{SiO}_{2}, \mathrm{Al}_{2} \mathrm{O}_{3}$ e $\mathrm{Fe}_{2} \mathrm{O}_{3}$, que são os compostos encontrados nos argilominerais caulinita, ilita e montmorilonita, característicos das argilas brasileiras utilizadas para cerâmica vermelha ou estrutural (Souza Santos, 1989). Com relação ao resíduo de corte de rochas ornamentais, se verifica que há maior quantidade de $\mathrm{SiO}_{2}$ e menores quantidades de $\mathrm{Al}_{2} \mathrm{O}_{3}$ e $\mathrm{Fe}_{2} \mathrm{O}_{3}$ quando comparado com as quantidades presentes na argila. A presença de maior quantidade de $\mathrm{SiO}_{2}, \mathrm{~K}_{2} \mathrm{O}, \mathrm{Na}_{2} \mathrm{O}, \mathrm{CaO}$ e $\mathrm{Al}_{2} \mathrm{O}_{3}$ é um indicativo de que o resíduo de corte de rochas ornamentais é proveniente principalmente de granitos, uma vez que o quartzo $\left(\mathrm{SiO}_{2}\right)$, o feldspato potássico $\left(\mathrm{KAlSi}_{3} \mathrm{O}_{8}\right)$ e a albita $\left(\mathrm{NaAlSi}_{3} \mathrm{O}_{8}\right)$ são minerais predominantes nessa rocha (Villas, 1999).

\section{Ensaios físicos}

Os resultados dos ensaios físicos encontram-se na Tabela 2. Os resultados referentes ao traço A50 não estão apresentados porque os corpos de prova não apresentaram boa qualidade durante prensagem (corpos de prova esfarelaram e/ou trincaram durante a confecção).

Tabela 2 - Resultados dos ensaios físicos dos corpos de prova sinterizados a $850^{\circ} \mathrm{C}, 900^{\circ} \mathrm{C}$ e $950^{\circ} \mathrm{C}$.

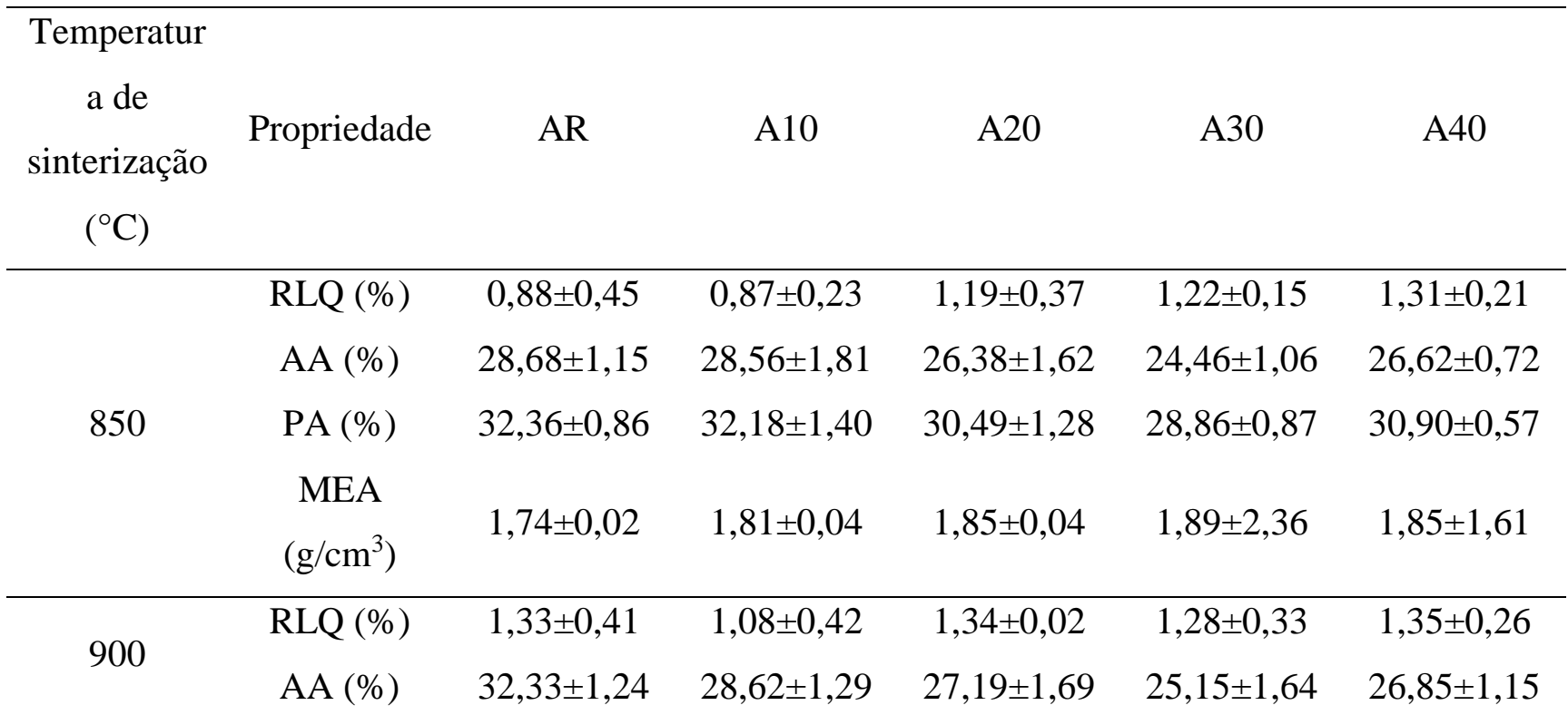




\begin{tabular}{ccccccc} 
& PA (\%) & $35,55 \pm 0,84$ & $32,78 \pm 0,99$ & $31,29 \pm 1,34$ & $29,76 \pm 1,30$ & $31,03 \pm 0,88$ \\
MEA & $1,77 \pm 0,03$ & $1,82 \pm 0,03$ & $1,84 \pm 0,04$ & $1,89 \pm 0,04$ & $1,85 \pm 0,02$ \\
$\left(\mathrm{~g} / \mathrm{cm}^{3}\right)$ & & & & & \\
\hline & RLQ (\%) & $1,55 \pm 0,31$ & $1,61 \pm 0,37$ & $1,37 \pm 0,01$ & $1,26 \pm 0,15$ & $1,38 \pm 0,18$ \\
& AA (\%) & $31,32 \pm 1,18$ & $29,36 \pm 1,94$ & $25,82 \pm 1,60$ & $25,09 \pm 1,44$ & $26,73 \pm 0,70$ \\
& PA (\%) & $35,19 \pm 1,18$ & $33,58 \pm 1,44$ & $30,83 \pm 1,31$ & $30,16 \pm 1,17$ & $31,47 \pm 0,56$ \\
& MEA & $1,81 \pm 0,02$ & $1,81 \pm 0,04$ & $1,89 \pm 0,04$ & $1,90 \pm 0,03$ & $1,86 \pm 0,02$ \\
& & & & & \\
\hline
\end{tabular}

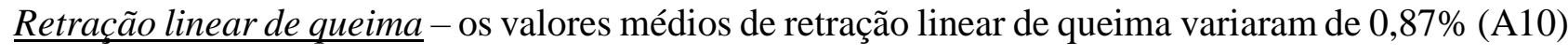
a $1,31 \%(\mathrm{~A} 40)$ a $850^{\circ} \mathrm{C}$; de $1,08 \%(\mathrm{~A} 10)$ a $1,35 \%(\mathrm{~A} 40)$ a $900^{\circ} \mathrm{C}$; e de $1,37 \%$ (A20) a $1,61 \%(\mathrm{~A} 10)$ a $950^{\circ} \mathrm{C}$.

Absorção de água - os valores médios de absorção de água variaram de 24,46\% (A30) a 28,56\% (A10) a $850^{\circ} \mathrm{C}$; de $25,15 \%$ (A30) a $32,33 \%(\mathrm{AR})$ a $900^{\circ} \mathrm{C}$; e de $25,09 \%$ (A30) a $31,32 \%$ (AR) a $950^{\circ} \mathrm{C}$.

Porosidade aparente - a porosidade aparente variou, em média, de 28,86\% (A30) a 32,36\% (AR) a $850^{\circ} \mathrm{C}$; de $29,76 \%$ (A30) a $35,55 \%$ (AR) a $900^{\circ} \mathrm{C}$; e de $30,16 \%\left(\mathrm{~A} 30\right.$ ) a $35,19 \%$ (AR) a $950^{\circ} \mathrm{C}$.

Massa específica aparente - os valores médios de massa específica aparente variaram de $1,74 \mathrm{~g} / \mathrm{cm}^{3}$ (AR) a $1,89 \mathrm{~g} / \mathrm{cm}^{3}$ (A30) a $850^{\circ} \mathrm{C}$; de $1,77 \mathrm{~g} / \mathrm{cm}^{3}$ (AR) a $1,89 \mathrm{~g} / \mathrm{cm}^{3}$ (A30) a $900^{\circ} \mathrm{C}$; e de $1,81 \mathrm{~g} / \mathrm{cm}^{3}$ (AR) a $1,90 \mathrm{~g} / \mathrm{cm}^{3}(\mathrm{~A} 30)$ a $950^{\circ} \mathrm{C}$.

As Figuras 1, 2, 3 e 4 ilustram graficamente a retração linear de queima, a absorção de água, a porosidade aparente e a massa específica aparente dos traços AR, A10, A20, A30 e A40 após sinterização a $850^{\circ} \mathrm{C}, 900^{\circ} \mathrm{C}$ e $950^{\circ} \mathrm{C}$, respectivamente, para melhor visualização do comportamento das propriedades físicas. 


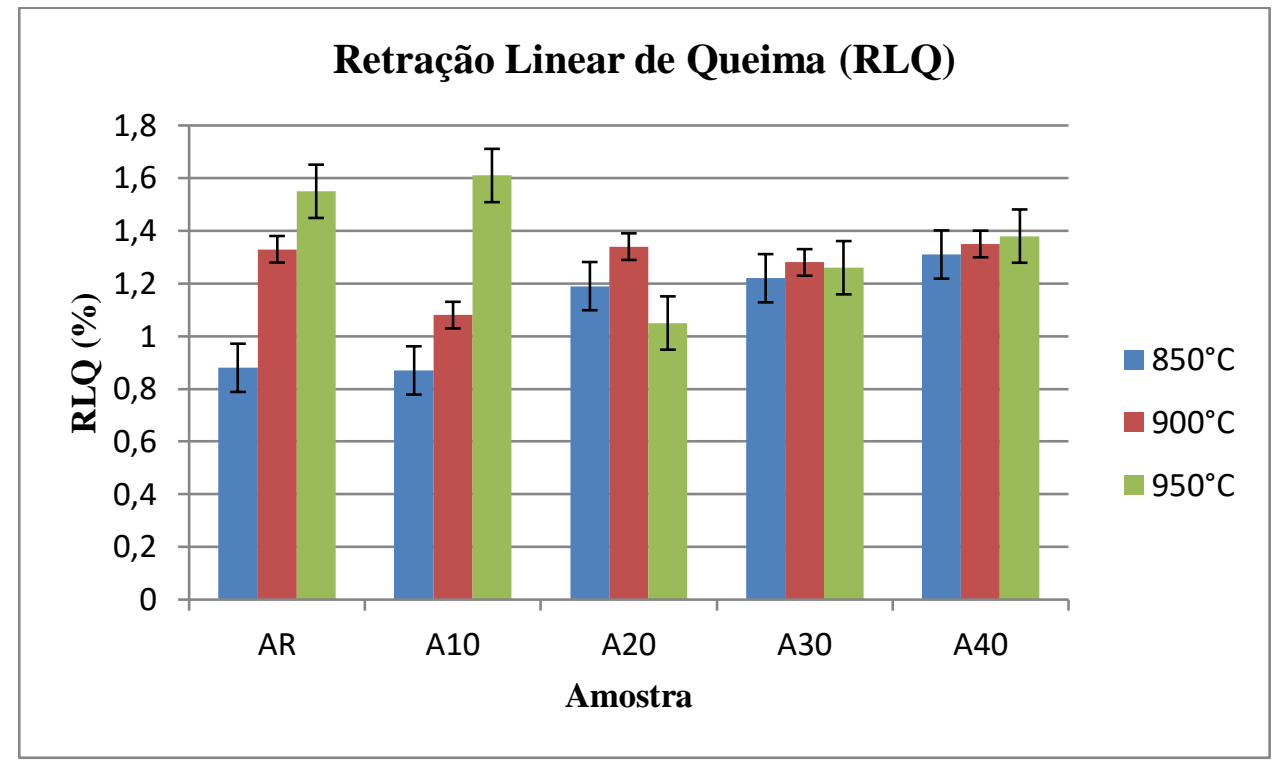

Figura 1 - Comportamento da retração linear de queima dos traços AR, A10, A20, A30 e A40 após sinterização a $850^{\circ} \mathrm{C}, 900^{\circ} \mathrm{C}$ e $950^{\circ} \mathrm{C}$.

Os traços A20, A30 e A40 apresentaram maior retração linear de queima quando comparados com os traços $\mathrm{AR}$ e $\mathrm{A} 10$ a $850^{\circ} \mathrm{C}$. Entretanto, a $900^{\circ} \mathrm{C}$ a retração linear de queima dos traços estudados foi praticamente a mesma (exceção para $\mathrm{A} 10$ ), enquanto que a $950^{\circ} \mathrm{C}$, a retração linear de queima dos traços A20, A30 e A40 foi menor que dos traços AR e A10.

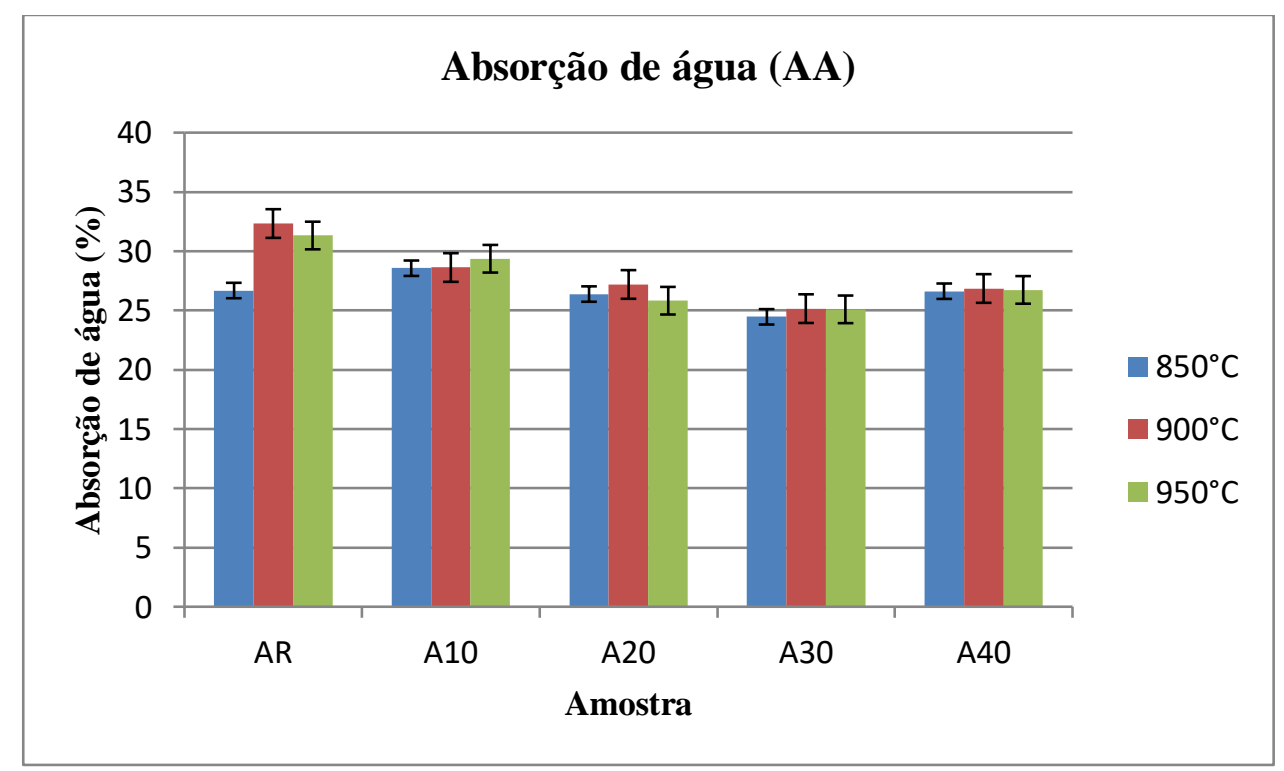

Figura 2 - Comportamento da absorção de água dos traços AR, A10, A20, A30 e A40 após sinterização a $850^{\circ} \mathrm{C}, 900^{\circ} \mathrm{C}$ e $950^{\circ} \mathrm{C}$.

Analisando-se cada traço individualmente, Observa-se que os traços com adição de resíduo de corte de rochas ornamentais possui comportamento semelhante, independente da temperatura de sinterização. De forma geral, os traços com resíduo de corte de rochas ornamentais apresentaram 
menor absorção de água por possuírem partículas menores que as da argila, diminuindo a porosidade dos corpos de prova.

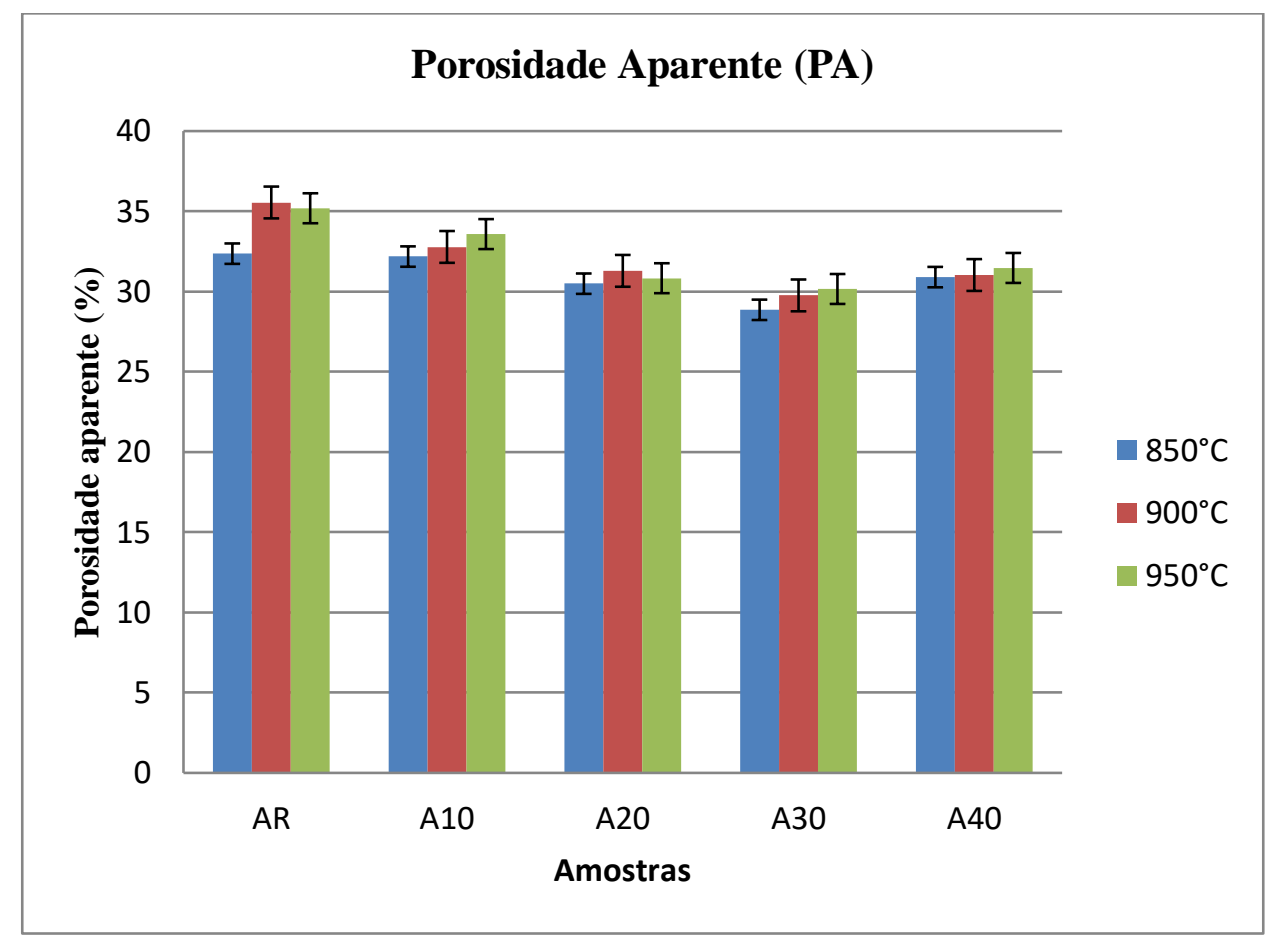

Figura 3 - Comportamento da porosidade aparente dos traços AR, A10, A20, A30 e A40 após sinterização a $850^{\circ} \mathrm{C}, 900^{\circ} \mathrm{C}$ e $950^{\circ} \mathrm{C}$.

Observa-se o mesmo comportamento de porosidade aparente para todos os traços estudados. Também é possível observar que, em maiores temperaturas de sinterização, os traços A20, A30 e A40 apresentaram menor porosidade aparente, enquanto os traços AR e A10 apresentaram comportamento semelhante. Esse comportamento pode ser explicado levando-se em conta que as partículas do resíduo são menores que as da argila, e que elas possivelmente se acomodaram nos interstícios das partículas da argila, reduzindo, assim, a porosidade aberta dos corpos de prova. 


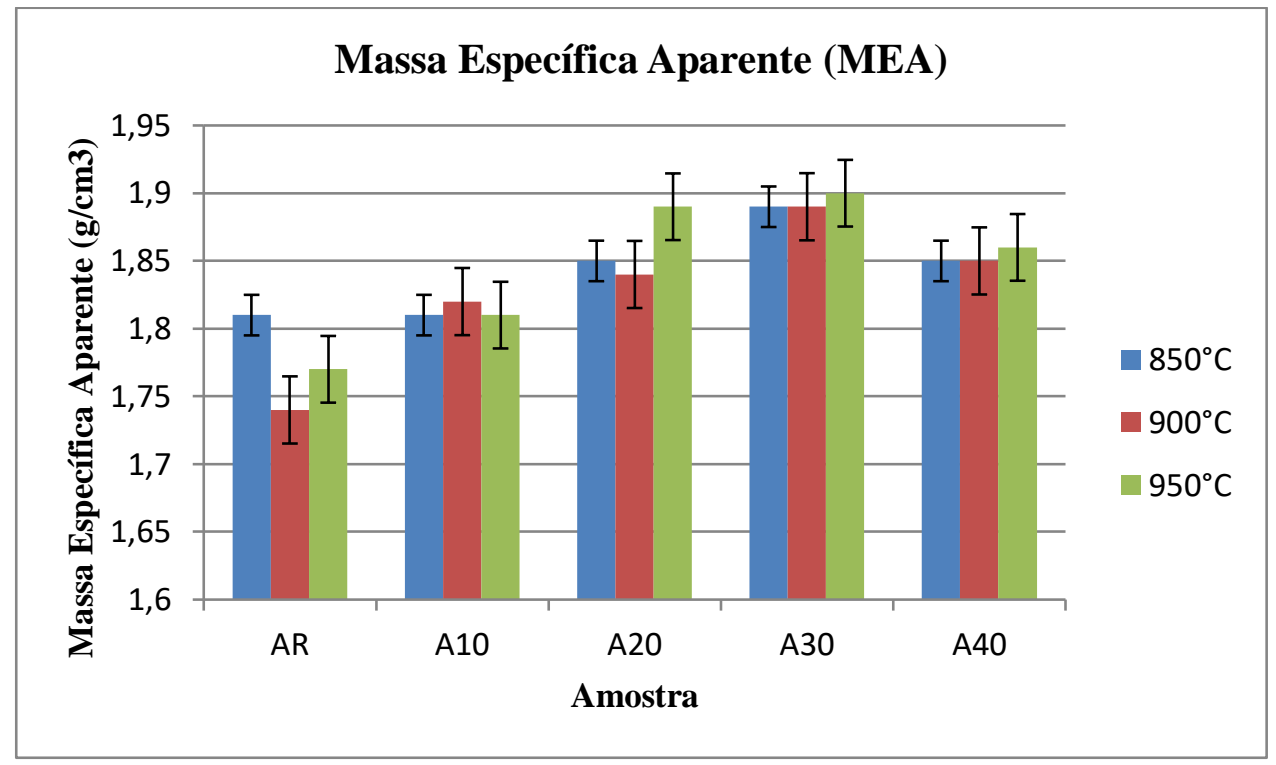

Figura 4 - Comportamento da massa específica aparente dos traços AR, A10, A20, A30 e A40 após sinterização a $850^{\circ} \mathrm{C}, 900^{\circ} \mathrm{C}$ e $950^{\circ} \mathrm{C}$.

Os resultados médios de massa específica aparente indicam que a incorporação do resíduo de corte de rochas ornamentais aumentou a densificação dos corpos de prova dos traços A10, A20, A30 e A40 quando comparados com os do traço AR. Esses resultados estão alinhados com os de absorção de água e porosidade aparente, uma vez que com menos poros abertos, menor absorção de água e maior densificação dos corpos de prova.

\section{Ensaios mecânicos}

Foram avaliados os limites de resistência à compressão (LRC) dos traços AR, A10, A20, A30 e A40 sinterizados a $850^{\circ} \mathrm{C}, 900^{\circ} \mathrm{C}$ e $950^{\circ} \mathrm{C}$. Os resultados estão ilustrados na Tabela 3. Os ensaios foram realizados a $24^{\circ} \mathrm{C}$ e umidade relativa de $48 \%$.

Tabela 3 - Resultados dos ensaios mecânicos dos corpos de prova sinterizados a $850^{\circ} \mathrm{C}, 900^{\circ} \mathrm{C}$ e $950^{\circ} \mathrm{C}$.

\begin{tabular}{ccccccc}
\hline $\begin{array}{c}\text { Temperatura } \\
\text { de } \\
\text { sinterização } \\
\left({ }^{\circ} \mathrm{C}\right)\end{array}$ & Propriedade & AR & A10 & A20 & A30 & A40 \\
\hline 850 & & & & & & \\
& & & & & & \\
900 & LRC (MPa) & $\pm 4,92$ & $24,32 \pm 3,86$ & $24,60 \pm 4,49$ & $24,08 \pm 4,25$ & $13,68 \pm 3,99$ \\
950 & & $24,24 \pm 3,04$ & $27,65 \pm 3,53$ & $24,94 \pm 4,54$ & $24,69 \pm 4,87$ & $14,27 \pm 3,03$ \\
& & $25,07 \pm 5,28$ & $26,49 \pm 4,61$ & $29,46 \pm 4,79$ & $27,82 \pm 4,19$ & $13,02 \pm 4,64$
\end{tabular}


Limite de resistência à compressão - o limite de resistência à compressão médio variou de 13,68 $\mathrm{MPa}(\mathrm{A} 40)$ a $32,68 \mathrm{MPa}(\mathrm{AR})$ a $850^{\circ} \mathrm{C}$; de $14,27 \mathrm{MPa}(\mathrm{A} 40)$ a $27,65 \mathrm{MPa}(\mathrm{A} 10)$ a $900^{\circ} \mathrm{C}$; e de 13,02 $\mathrm{MPa}(\mathrm{A} 40)$ a $29,46 \mathrm{MPa}(\mathrm{A} 20)$ a $950^{\circ} \mathrm{C}$.

A Figura 5 ilustra o comportamento do limite de resistência à compressão dos traços AR, A10, A20, A30 e A40 após sinterização a $850^{\circ} \mathrm{C}, 900^{\circ} \mathrm{C}$ e $950^{\circ} \mathrm{C}$.

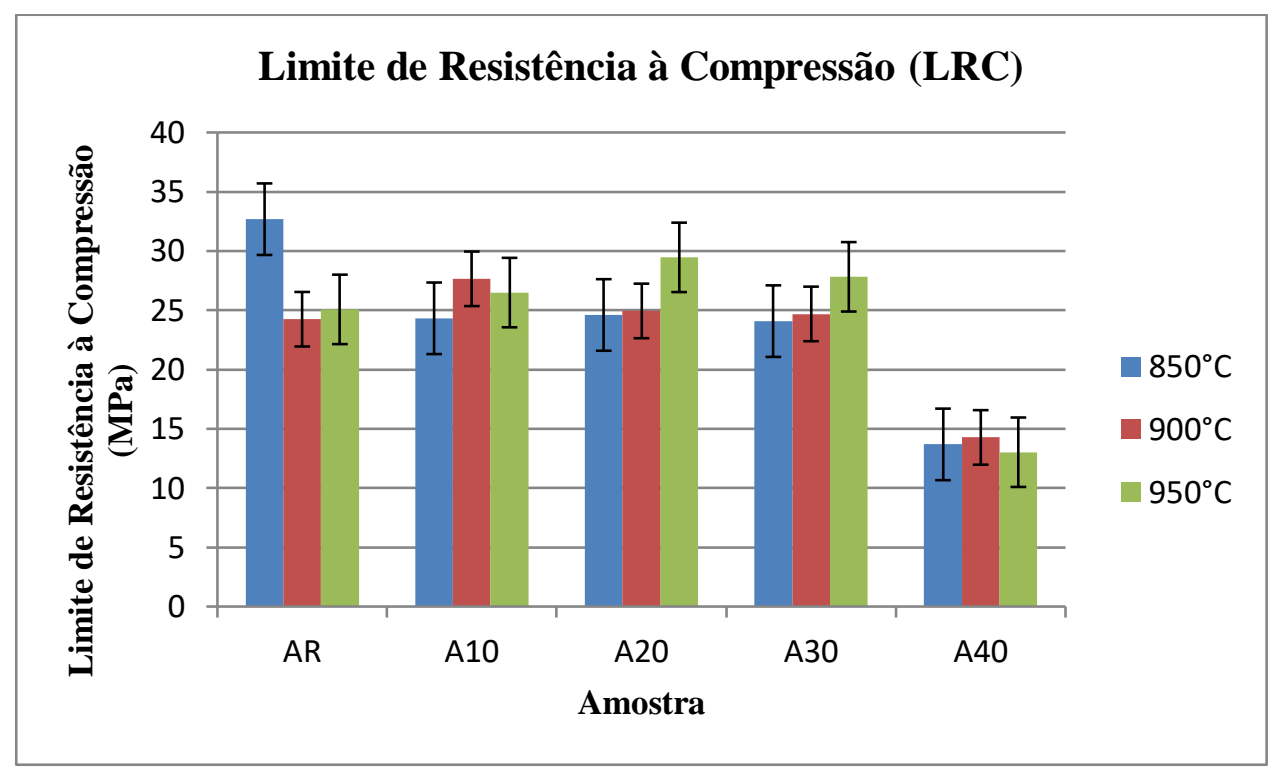

Figura 5 - Comportamento do limite de resistência à compressão dos traços AR, A10, A20, A30 e A40 após sinterização a $850^{\circ} \mathrm{C}, 900^{\circ} \mathrm{C}$ e $950^{\circ} \mathrm{C}$.

Observa-se que com o aumento da temperatura de sinterização, o limite de resistência à compressão do traço AR e dos traços A10, A20 e A30 praticamente são os mesmos. A exceção é o traço A40, que apresentou valores inferiores aos demais traços.

Uma observação que se faz é que os corpos de prova do traço A40 apresentaram aspecto arenoso, onde ao se tocar nos corpos de prova foi possível observar que partículas aderiram aos dedos, fato não apresentado nos corpos de prova dos demais traços.

\section{CONCLUSÕES}

Foram avaliadas as propriedades físicas e mecânicas de massas cerâmicas com 0\%, 10\%, 20\%, 30\%, $40 \%$ e $50 \%$ em massa de resíduo de corte de rochas ornamentais sinterizadas a $850^{\circ} \mathrm{C}, 900^{\circ} \mathrm{C}$ e $950^{\circ} \mathrm{C}$ a fim de estabelecer a possibilidade de utilização desse resíduo em massas cerâmicas para produtos cerâmicos estruturais. 
Os traços com adição de resíduo de corte de rochas ornamentais apresentaram, de forma geral, menor absorção de água, menor porosidade aparente e maior massa específica aparente quando comparados com o traço de referência, o que significa que esses traços são mais densos que o traço de referência.

Os corpos de prova dos traços A20, A30 e A40 tiveram maior retração linear de queima a $850^{\circ} \mathrm{C}$, praticamente a mesma retração a $900^{\circ} \mathrm{C}$ e retração menor a $950^{\circ} \mathrm{C}$ quando comparados aos traços $\mathrm{AR}$ e A10. Esse comportamento pode estar relacionado com a maior quantidade de $\mathrm{SiO}_{2}$ e a menor quantidade de $\mathrm{Al}_{2} \mathrm{O}_{3}$ presentes no resíduo.

O limite de resistência à compressão para os traços estudados apresentaram valores similares à medida que a temperatura de sinterização foi aumentada; porém, essa propriedade foi inferior para o traço A40 quando comparado com os demais traços.

De forma geral, pode-se concluir que é possível adicionar até 30\% em massa de resíduo de corte de rochas ornamentais sem comprometer as propriedades físicas e mecânicas das massas cerâmicas utilizadas na indústria de cerâmica estrutural.

\section{AGRADECIMENTOS}

Os autores gostariam de agradecer à Cerâmica Lubiana Ltda. e ao Centro de Tratamento de Resíduo Nova Venécia S/A pela doação das matérias primas utilizadas nesse trabalho e ao Instituto Federal do Espírito Santo pela bolsa de iniciação científica e pelo auxílio para apresentação do trabalho no $60^{\circ}$ Congresso Brasileiro de Cerâmica.

\section{REFERÊNCIAS}

ALMEIDA, N.; BRANCO, F.; BRITO, J.; SANTOS, J. R. High-performance concrete with recycled stone slurry. Cement and Concrete Research, v.37, p. 210-220. 2007.

ALMEIDA, N.; BRANCO, F.; SANTOS, J. R. Recycling of stone slurry in industrial activities: Application to concrete mixtures. Building and Environment, v. 42, p. 810-819. 2007.

COSTA, A. S. V.; HORN, A. H.; DONAGEMMA, G. K.; SILVA, M. B. Uso do resíduo de granito oriundo da serraria e polimento como corretivo e fertilizante de solos agrícolas. Geonomos, v. 18, p. 23-27. 2010.

ELIMBI, A.; NJOPWOUO, D. Potential of using granite waste as raw material for geopolymer synthesis. Ceramics International, v. 42, p. 3046-3055. 2016.

GHANNAMA, S.; NAJM, H.; VASCONEZ, R. Experimental study of concrete made with granite and iron powders as partial replacement of sand. Sustainable Materials and Technologies, v. 9, p. 1-9. 2016. 
HOJAMBERDIEV, M.; EMINOV, A.; XU, Y. Utilization of muscovite granite waste in the manufacture of ceramic tiles. Ceramics International, v. 37, p. 871-876. 2011.

KANG, J.; WANG, J.; CHENG, J.; YUAN, J.; HOU, Y.; QIAN, S. Crystallization behavior and properties of $\mathrm{CaO}-\mathrm{MgO}-\mathrm{Al}_{2} \mathrm{O}_{3}-\mathrm{SiO}_{2}$ glass-ceramics synthesized from granite wastes. Journal of Non-Crystalline Solids, v. 457, p. 111-115, 2017.

LIMA, W. B. C.; LIRA, H. L.; NEVES, G. A. Incorporação de resíduo lama do processo de beneficiamento de granito na argamassa expansiva de demolição. Revista Eletrônica de Materiais e Processos, v. 11, n. 2, p. 89-95, 2016.

LOKESHWARI, M.; JAGADISH, K. S. Eco-Friendly use of Granite Fines Waste in Building Blocks. Procedia Environmental Sciences, v. 35, p. 618 - 623. 2016.

MÁRMOL, I.; BALLESTER, P.; CERRO, S.; MONROS, G.; MORALES, J.; SANCHEZ, L. Use of granite sludge wastes for the production of coloured cement-based mortars. Cement \& Concrete Composites, v. 32, p. 617-622. 2010.

MEDINA, G.; SÁEZ DEL BOSQUE, I. F.; FRÍAS, M.; SÁNCHEZ DE ROJAS, M. I.; MEDINA, C. Mineralogical study of granite waste in a pozzolan/Ca $(\mathrm{OH})_{2}$ system: Influence of the activation process. Applied Clay Science, v. 135, p. 362-371. 2017.

MENEZES, R. R.; FARIAS FILHO, J.; FERREIRA, H. S.; NEVES, G. A.; FERREIRA, H. C. Reciclagem de resíduos da construção civil para a produção de argamassas. Cerâmica, v. 55, p. 263270. 2009.

RANA, A.; KALLA, P.; VERMA, H. K.; MOHNOT, J. K. Recycling of dimensional stone waste in concrete: A review. Journal of Cleaner Production, v. 135, p. 312-331. 2016.

SADEK, D. M.; EL-ATTAR, M. M.; ALI, H. A. Reusing of marble and granite powders in selfcompacting concrete for sustainable development. Journal of Cleaner Production, v. 121, p. 1932. 2016.

SARDOU FILHO, R.; MATOS, G. M. M.; MENDES, V. A.; IZA, E. R. H. F. Atlas de rochas ornamentais do estado do Espírito Santo, Brasília: CPRM (2013) 17.

SINGH, S.; NAGAR, R.; AGRAWAL, V. Performance of granite cutting waste concrete under adverse exposure conditions. Journal of Cleaner Production, v. 127, p. 172-182. 2016a.

SINGH, S.; NAGAR, R.; AGRAWAL, V. A review on Properties of Sustainable Concrete using granite dust as replacement for river sand. Journal of Cleaner Production, v. 126, p. 74 - 87. 2016 b.

SINGH, S.; NAGAR, R.; AGRAWAL, V.; RANA, A.; TIWARI, A. Sustainable utilization of granite cutting waste in high strength concrete. Journal of Cleaner Production, v. 116, p. 223 - 235. 2016 a.

SINGH, S.; KHAN, S.; KHANDELWAL, R.; CHUGH, A.; NAGAR, R. Performance of sustainable concrete containing granite cutting waste. Journal of Cleaner Production, v. 119, p. 86 - 98. 2016 b. 
SINGH, S.; TIWARI, A.; NAGAR, R.; AGRAWAL, V. Feasibility as a Potential Substitute for Natural Sand: A Comparative Study between Granite Cutting Waste and Marble Slurry Procedia Environmental Sciences, 35 (2016c) $571-582$.

SOLTAN, A. M. M.; KAHL, W. A.; RAOOF, F. A.; EL-KALIOUBY, B. A. H.; SERRY, M. A. K.; ABDEL-KADER, N. A. Lightweight aggregates from mixtures of granite wastes with clay. Journal of Cleaner Production, v. 117, p. 139-149. 2016.

SOUZA, L. G. M.; SANTOS, N. R.; CAVALCANTE, A. G.; SOUZA, L. G. V. M.; COSTA, L. C. F. Composite utilizing residues of marble and granite for building popular homes. Journal of Building Engineering, v. 9, p. 192-197. 2017.

SOUZA SANTOS, P. Ciência e tecnologia de argilas - Vol. 1. $2^{\mathrm{a}}$ ed. São Paulo: Edgard Blucher Ltda., 1989, 175 - 209; 393 - 408.

TCHADJIÉ, L. N.; DJOBO, J. N. Y.; RANJBAR, N.; TCHAKOUTÉ, H. K.; KENNE, B. B. D.; LI, Y.; YU, H.; ZHENG, L.; WEN, J.; WU, C.; TAN, Y. Compressive strength of fly ash magnesium oxychloride cement containing granite wastes. Construction and Building Materials, v. 38, p. 1-7. 2013.

TORRES, P.; FERNANDES, H. R.; OLHERO, S.; FERREIRA, J. M. F. Incorporation of wastes from granite rock cutting and polishing industries to produce roof tiles. Journal of the European Ceramic Society, v. 29, p. 23-30. 2009.

VIEIRA, C. M. F.; SOARES, T. M.; SÁNCHEZ, R.; MONTEIRO, S. N. Incorporation of granite waste in red ceramics. Materials Science and Engineering A, v. 373, p. 115-121. 2004.

VIJAYALAKSHMI, M.; SEKAR, A. S. S.; GANESH PRABHU, G. Strength and durability properties of concrete made with granite industry waste. Construction and Building Materials, v. 46, (2013) p. 1-7. 2013.

VILLAS, R. N. Granito Pojuca, serra dos Carajás (PA): composição mineralógica, química mineral e controles químicos da alteração hidrotermal. Revista Brasileira de Geociências, v. 29, n. 3, p. 393404. 1999. 